\begin{tabular}{|c|c|c|}
\hline $\begin{array}{l}\text { PKS } \\
\text { PUBLIC } \\
\text { KNOWLEDGE } \\
\text { PROJECT }\end{array}$ & $\begin{array}{c}\text { REVISTA DE GEOGRAFIA } \\
\text { (RECIFE) } \\
\text { http://www.revista.ufpe.br/revistageografia }\end{array}$ & $\begin{array}{l}\text { OJS } \\
\begin{array}{l}\text { OPEN } \\
\text { JOUNAL } \\
\text { SYSTEMS }\end{array}\end{array}$ \\
\hline
\end{tabular}

\title{
CORRELAÇÃO ENTRE A PRECIPITAÇÃO E TEMPERATURA MÉDIA DO AR SOBRE O NÚMERO DE REGISTROS DE CASOS DE DENGUE PARA CIDADES DE MACEIÓ, JOÃO PESSOA E SALVADOR
}

\author{
Osmar Evandro Toledo Bonfim ${ }^{1}$, Ítalo Ramon Januário ${ }^{2 .}$
}

\begin{abstract}
${ }^{1}$ Mestre em Meteorologia pelo Instituto de Ciências Atmosféricas - ICAT/UFAL. Email:osmartoledob@gmail.com

${ }^{2}$ Mestre em Meteorologia pelo Instituto de Ciências Atmosféricas - ICAT/UFAL. E-mail: italojanuario@hotmail.com
\end{abstract}

Artigo recebido em 15/08/2017 e aceito em 25/08/2018

\begin{abstract}
RESUMO
A dengue é uma doença que afeta milhares de pessoas que residem nas regiões de clima tropical e subtropical. Em épocas do ano mais quentes e úmidas ocorrem em todo mundo o aumento da incidência da doença. Objetivou-se neste trabalho estudar a relação das variáveis precipitação e temperatura média do ar com o número de casos de dengue. Além disto, simultaneamente foram calculadas as taxas de incidência da dengue para as três cidades nordestinas. Foram utilizados dados de precipitação e temperatura média do ar, assim como dados de registro de casos de dengue para o período de 2008 a 2015. A precipitação obteve uma boa correlação com os registros de casos de dengue nas três cidades, coincidindo um aumento nos registros da doença com o período chuvoso da região. Entretanto, houve correlação desprezível entre temperatura média do ar e o número de casos de dengue, tendo em vista que a região apresentou temperatura média favorável ao desenvolvimento do ciclo de vida do mosquito a qualquer época do ano. De um modo geral, os resultados apontam que as políticas de vigilância e combate ao foco do mosquito devem ser contínuas todos os meses do ano, não apenas no período chuvoso, a fim de reduzir os riscos de epidemias na região.
\end{abstract}

Palavras-chave: Aedes aegypti; Condições Climáticas; Incidência de Dengue.

\section{CORRELATION BETWEEN PRECIPITATION AND AVERAGE AIR TEMPERATURE OVER THE NUMBER OF DENGUE CASES REPORTED IN THE MACEIÓ, JOÃO PESSOA AND SALVADOR CITIES}

\begin{abstract}
Dengue is a disease who affects mostly of people living in tropical and subtropical regions. At warmer and hotter times of the year, the incidence of the disease increase worldwide. The purpose of this study is to evaluate the relationship between variables precipitation and average air temperature with the reported cases of dengue variation. In addition, the coefficients of dengue incidence for the three norhteastern cities were calculated. There were used precipitation and average air temperature data of three Brazilian Northeastern capitals, as well as dengue case data with period from 2008 to 2015 . The precipitation variable obtained a good correlation with the records of dengue cases reported in the three cities, an increase in the records of the disease coincided with the rainy season of the region. However, there was no association between average air temperature and the number of dengue cases, considering that the region presented average temperatures favorable to the development of the mosquito life cycle at any time of the year. Overall, the results indicate that surveillance and mosquito control policies should be accomplish every month of the year, not just during the rainy season, in order to reduce the risk of epidemics in the region.
\end{abstract}

Keywords: Aedes aegypti; Climate conditions; Dengue incidence. 


\section{INTRODUÇÃO}

A dengue é uma arbovirose transmitida ao homem pela picada de mosquitos fêmeas do gênero Aedes, sendo o Aedes aegypti o vetor mais importante da doença. A dengue se tornou ao longo das últimas décadas um grande problema de saúde pública mundial, proporcionando risco a quase quatro bilhões de pessoas que vivem em regiões de clima tropical e subtropical do mundo, predominantemente em áreas urbanas e semiurbanas (ARAUJO et al., 2017; FERREIRA et al., 2018).

No Brasil, a partir da década de 90, houve um notável aumento no número de casos de dengue. Segundo os dados do Ministério da Saúde, no ano de 2016, foram registrados mais de 1.000.000 de casos prováveis da doença, colocando o país com o maior detentor de número de casos de dengue em toda a América do Sul (BRASIL, 2018).

A dengue no Brasil apresenta um padrão sazonal, com maior incidência de casos nos primeiros cinco meses do ano, período mais quente e úmido, típico dos climas tropicais. Este tipo de clima, presente na maior parte do país, favorece a proliferação do mosquito Aedes aegypti (BRAGA \& VALLE, 2007).

O ministério da Saúde utiliza a taxa de incidência de dengue para analisar as variações geográficas e temporais na distribuição dos casos confirmados de dengue. Segundo Santos et al. (2016), a possível falta de saneamento adequado nas cidades influencia na densidade de mosquitos e na incidência de dengue.

A região Nordeste do Brasil (NEB) atualmente apresenta um dos maiores índices de casos de dengue e também óbitos por febre hemorrágica de dengue (FHD) no país (BRASIL, 2018). As cidades de Maceió, João Pessoa e Salvador pertencentes à costa Leste do Nordeste, apresentam temperaturas elevadas durante o ano todo e regime pluviométrico concentrado entre os meses de abril a agosto. Este clima torna-se favorável ao desenvolvimento do vetor da dengue praticamente a qualquer época do ano (BESERRA et al., 2006).

Sabendo que o aumento na temperatura, precipitação e variações na umidade relativa do ar propicia o número de criadouros disponível e o desenvolvimento do vetor (VIANA \& IGNOTTI, 2013). Um estudo sobre a influência dessas variáveis no aumento de casos de dengue torna-se importante para os órgãos públicos e a população, a fim de adotar medidas satisfatórias nos processos de planejamento, gestão e avaliação de políticas e ações de saúde direcionadas para controlar a dengue.

O objetivo deste estudo visa averiguar a correlação da precipitação e da temperatura média do ar na variação de casos de dengue para as cidades de Maceió, João Pessoa e Salvador. 


\section{METODOLOGIA}

Maceió, capital do estado de Alagoas localizado na costa Leste do NEB, situa-se entre o oceano Atlântico e a Lagoa Mundaú entre as coordenadas geográficas 9³9'57’ latitude sul e $35^{\circ} 44^{\prime} 07^{\prime}$ ' longitude oeste. O Clima é do tipo quente e úmido, caracterizado por não apresentar grandes diferenciações térmicas, os maiores índices pluviométricos ocorrem entre abril e julho (ARAUJO et al. 2009). A população estimada em 2015 pelo Instituto Brasileiro de Geografia e Estatística (IBGE, 2017) é de 1.013.773 habitantes.

João Pessoa, capital do estado da Paraíba, localiza-se no Leste do NEB próximo a ponta do Seixas, conhecido como ponto mais oriental das Américas, cujas coordenadas geográficas são $7^{\circ} 07^{\prime} 28^{\prime \prime}$ latitude sul e 3453'30” longitude oeste. O clima de João pessoa é do tipo tropical úmido, com chuvas em grandes quantidades praticamente o ano todo (DANTAS et al., 2007). Segundo IBGE (2017), João Pessoa possui uma população estimada de 801.718 habitantes.

A cidade de Salvador localiza-se na latitude $12^{\circ} 58^{\prime} 16^{\prime \prime}$ sul e longitude $38^{\circ} 30^{\prime} 39^{\prime \prime}$ oeste próximo a Zona da Mata no Leste do NEB. De acordo com Barbosa et al. (2003), o clima é quente e úmido e a temperatura média anual apresenta-se em torno de $25^{\circ} \mathrm{C}$. $\mathrm{O}$ índice pluviométrico de Salvador é superior a $2000 \mathrm{~mm} / \mathrm{ano}$, sendo maio o mês de maior precipitação. Segundo o IBGE (2017) a população do município é cerca de 2.938 .032 habitantes.

Neste trabalho foram utilizados dados de precipitação e temperatura média do ar mensal para o período de 2008 a 2015 dos três municípios em estudo. Os dados foram obtidos através do Banco de Dados Meteorológico de Ensino e Pesquisa (BDMEP) do Instituto Nacional de Meteorologia (INMET). Também foram utilizados dados de números de casos notificados de dengue do banco de dados de informações de Saúde (TABNET) disponível no Departamento de informática do Sistema Único de Saúde (DATASUS).

É importante ressaltar que o período selecionado para os dados de precipitação e temperatura média do ar esteve condicionado ao período selecionado para os casos notificados de dengue.

A taxa de incidência de dengue foi calculada para todo o período, no qual considera o número de casos da doença dividido pela população exposta, multiplicado 100 mil habitantes, classificando o resultado de acordo com a escala adotada pelo Programa Nacional de Controle da Dengue descrito na Tabela 01 . O cálculo da incidência é a forma mais comum de medir e comparar a frequência das doenças em populações (BRASIL, 2018). 
Tabela 01- Interpretação da taxa de incidência de dengue. Fonte: Brasil (2018)

\section{Taxa de incidência de dengue}

\begin{tabular}{cc}
\hline Baixa incidência & 100 casos por 100 mil hab. \\
Média incidência & 101 a 299 casos por 100 mil hab. \\
Alta incidência & Acima de 300 casos por 100 mil hab. \\
\hline
\end{tabular}

O tamanho da população empregado como denominador para o cálculo da incidência da dengue, teve como fonte os Censos Demográficos de 2000 e 2010. Para os anos intercensitários, foram utilizadas as projeções e estimativas populacionais disponíveis no portal do IBGE (https://ww2.ibge.gov.br/home/estatistica/ populacao/estimativa2015/estimativa_dou.shtm) (RIBEIRO et al., 2006; VALADARES et al., 2013).

A correlação de Pearson foi utilizada para analisar a associação entre a precipitação média e temperatura média em relação ao número de casos de dengue, visando identificar um padrão de variação conjunta entre essas variáveis. O coeficiente de correlação de Pearson (r) ou o r de Pearson mede o grau da correlação linear entre duas variáveis quantitativas (Tabela 02). É um índice adimensional com valores situados entre $-1,0$ e 1.0 que reflete a intensidade de uma relação linear entre dois conjuntos de dados (CÂMARA et al. 2007; VALADARES et al., 2013).

Tabela 02- Interpretação do coeficiente de correlação de Pearson

\begin{tabular}{ccccc}
\hline \multicolumn{4}{c}{ Coeficiente de correlação Linear de Pearson } \\
\hline Correlação muito forte & $>0.9$ & Correlação Inversa muito forte & $<-0,9$ \\
Correlação forte & 0,7 a 0,9 & Correlação inversa forte & $-0,9$ a 0,7 \\
Correlação moderada & 0,5 a 0,7 & Correlação inversa moderada & $-0,7$ a $-0,5$ \\
Correlação fraca & 0,3 a 0,5 & Correlação Inversa fraca & $-0,5$ a $-0,3$ \\
Correlação desprezível & 0 a 0,3 & Correlação inversa desprezível & $-0,3$ a 0 \\
\hline
\end{tabular}

O coeficiente de correlação de Pearson é normalmente representado pela letra $\mathbf{r}$ e a sua fórmula de cálculo é:

$$
r=\frac{\sum_{i}\left(x_{i}-\bar{x}\right)\left(y_{i}-\bar{y}\right)}{\sqrt{\sum_{i}\left(x_{i}-\bar{x}\right)^{2} \sum_{i}\left(y_{i}-\bar{y}\right)^{2}}}
$$

Em que $\mathrm{x} 1, \mathrm{x} 2 \ldots \mathrm{xN}$ e y1, y2... yN são os valores medidos de ambas as variáveis. 


\section{RESULTADOS E DISCUSSÕES}

\section{Precipitação pluviométrica média e o número médio de casos de dengue}

Para a cidade de Maceió notou-se um relação direta entre o número de casos de dengue registrados e o regime pluviométrico médio mensal no período de 2008 a 2015 (Figura 01). O mês de maio apresentou a maior média de registro de casos de dengue, que coincide com o período da quadra chuvosa (abril a julho) de Maceió, meses em que anualmente apresentam os maiores volumes de chuvas, respaldando os resultados encontrados por Flexa et al. (2008) e Costa et al. (2011), Costa \& Calado (2016).

Os meses entre outubro e janeiro representam o período de seca da região, onde há baixo índice de precipitação e por consequência houve uma redução gradual nos registros de casos de dengue. O mês de dezembro apresentou a menor média de registros de casos dengue como também o menor índice pluviométrico para o período de estudo.

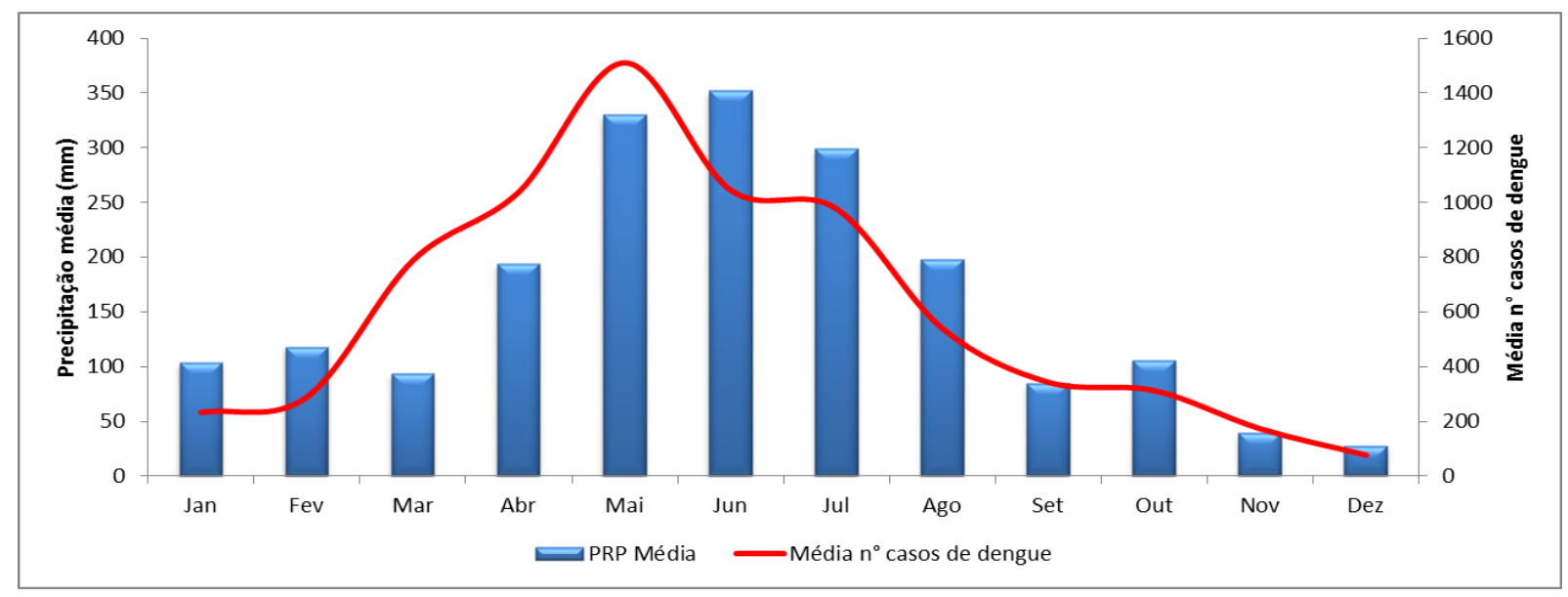

Figura 01- Relação entre a precipitação média e a média de n casos de dengue em Maceió (2008-2015).

$\mathrm{Na}$ análise da relação da precipitação média com os registros de casos de dengue em João Pessoa (Figura 02), notou-se um aumento no número de casos de dengue a partir do mês de março, porém no mês de junho, que representa o mês com maior precipitação da região, houve um declínio nos casos de dengue se estendendo até o período seco (setembro a dezembro), onde há baixos índices pluviométricos. 


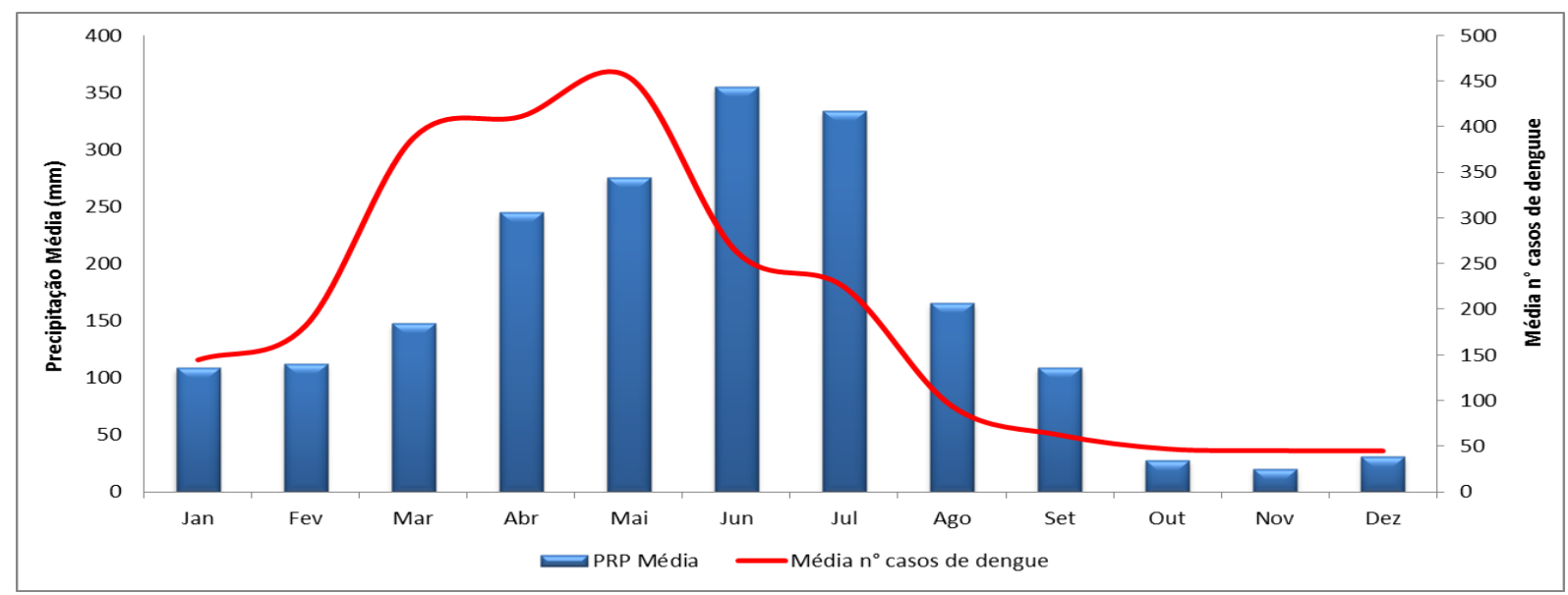

Figura 02- Relação entre a precipitação média e a média nº de casos de dengue em João Pessoa (2008-2015).

O mês de maio apresentou a maior média de registros de casos de dengue coincidindo com o período chuvoso de João Pessoa. O mês de novembro registrou a menor média no número de casos de dengue como também a menor média pluviométrica.

A relação entre o número de casos de dengue registrados e o regime médio de precipitação para Salvador se mostrou bem definido (Figura 03). O mês de maio apresentou a maior média de número de casos de dengue registrado, como também a maior média pluviométrica. Notou-se um aumento nos registros de casos de dengue no período chuvoso, exceto o mês de março que apresentou uma média alta de notificação de casos de dengue, porém uma média pluviométrica relativamente baixa.

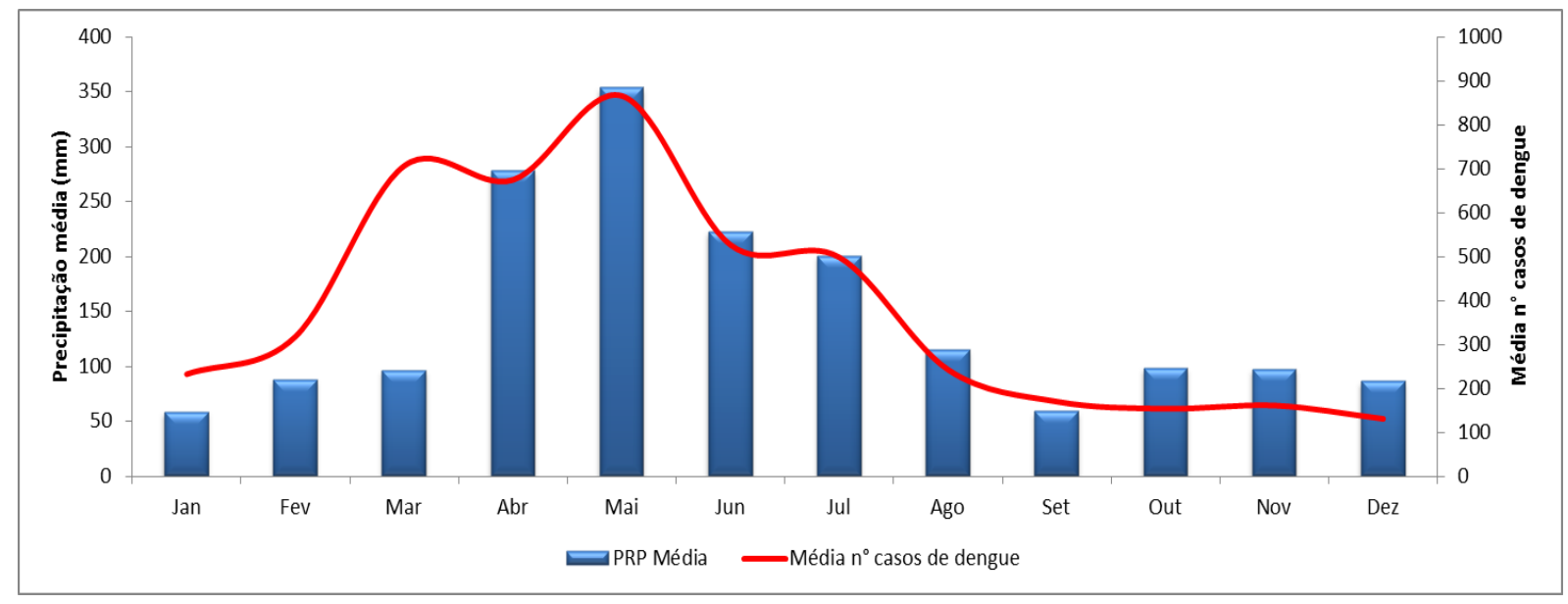

Figura 03- Relação entre a precipitação média e a média nº de casos de dengue em Salvador (2008-2015).

Os meses entre setembro e janeiro apresentaram redução na precipitação, neste período, o número de casos de dengue similarmente reduziu. O mês de dezembro foi o mês que registrou a menor média de notificação de casos dengue. A relação entre essas variáveis 
corrobora com os resultados encontrados por Sousa et al. (2006), Flexa et al. (2008) Costa et al. (2011), Costa \& Calado (2016).

Temperatura média do ar e o número médio de casos de dengue

$\mathrm{Na}$ análise da relação da temperatura média do ar com os registros de casos de dengue em Maceió (Figura 04), não foi possível observar uma estrutura lógica do número de casos de dengue com a variação mensal da temperatura da cidade de Maceió. Os meses entre junho a agosto exibiram as menores médias de temperatura do ar, no qual ocorreu o início do declínio nos registros de casos de dengue. Os meses seguintes (outubro a dezembro) as temperaturas se apresentaram mais elevadas, entretanto não houve aumento no registro da doença.

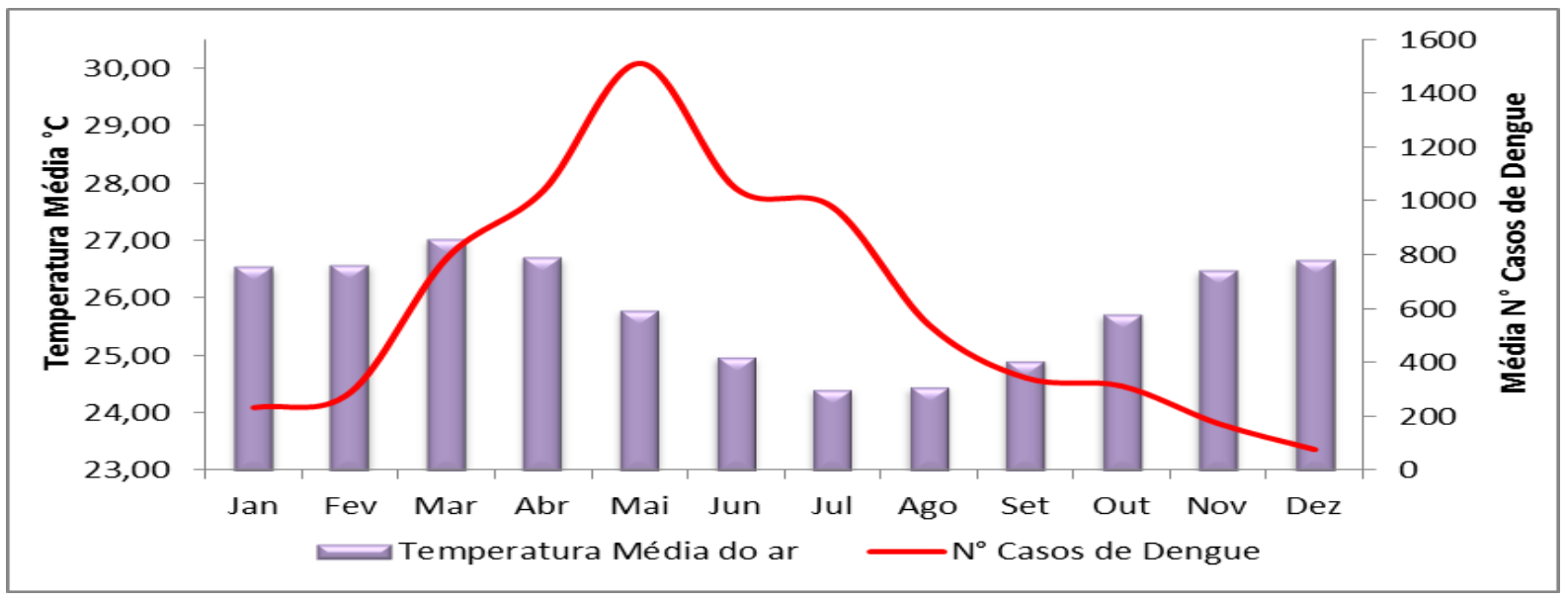

Figura 04- Relação entre a Temperatura Média do ar e a média nº de casos de dengue em Maceió (2008-2015).

Resultados semelhantes foram encontrados para a cidade de João Pessoa (Figura 05) e Salvador (Figura 06), as menores temperaturas médias se apresentaram entre os meses de maio e agosto, no qual houve um declínio no registro de casos de dengue neste período. Todavia não ocorreu um aumento de notificações de casos de dengue acompanhando os meses de verão, respaldando os resultados encontrados por Ferreira et al. (2018) . Assim como Maceió, temperatura média do ar de João Pessoa e de Salvador não apresentou grandes variações ao longo do ano, oscilando entre $25^{\circ} \mathrm{C}$ a $28^{\circ} \mathrm{C}$. 


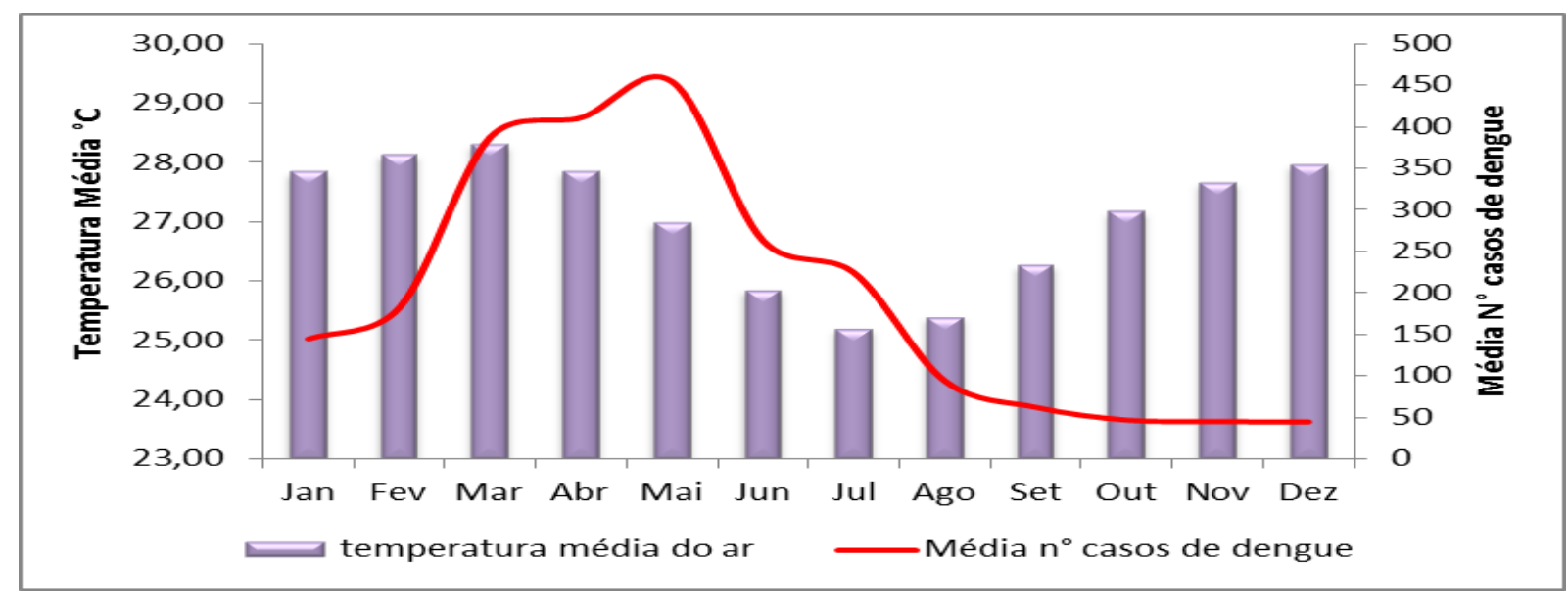

Figura 05- Relação entre a temperatura média do ar e a média de n de casos de dengue em João Pessoa (20082015).

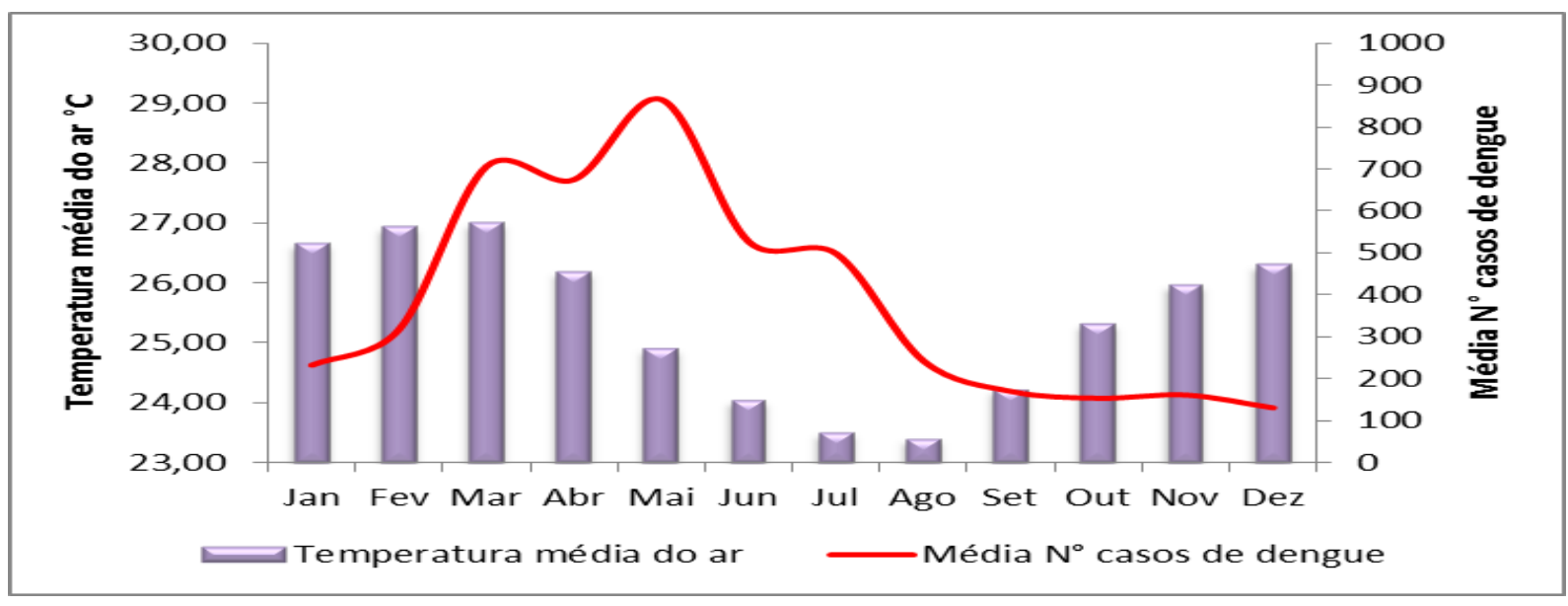

Figura 06 - Relação entre a Temperatura Média do ar e a Média nº de casos de dengue em Salvador (20082015).

Os gráficos de temperatura média do ar das três cidades em estudos não apresentaram grandes variações ao longo do ano, oscilando entre $22^{\circ} \mathrm{C}$ a $29^{\circ} \mathrm{C}$, o qual corresponde às condições favoráveis de temperatura para o desenvolvimento do ciclo biológico do mosquito (PACHECO et al., 2017) . Neste caso, apesar da temperatura média do ar não ter influenciado diretamente o aumento nos registros de casos de dengue, a região em estudo apresenta as condições ideais para a reprodução do vetor e a consequente proliferação dos casos de dengue em qualquer época do ano.

\section{Coeficiente de Correlação de Pearson}

No cálculo do coeficiente de correlação entre a precipitação e os registros de casos de dengue para Maceió, obteve-se um valor de $\rho=0,86$ (Correlação Forte), porém não houve 
correlação significativa entre a temperatura média do ar e o número de casos de dengue, apresentando um valor de $\rho=-0,25$ (Correlação inversa desprezível).

Para a cidade João Pessoa, obteve-se um valor de $\rho=0,68$ (Correlação Moderada) entre a precipitação e o número de casos de dengue, porém a correlação entre a temperatura média do ar e as notificações de casos de dengue não apresentou significância, obtendo um valor de $\rho=0,12$ (Correlação desprezível).

A correlação entre a precipitação e o número de casos de dengue para Salvador apresentou um valor de $\rho=0,81$ (Correlação Forte), entretanto não houve correlação significativa entre a temperatura média do ar e o número de registro de casos de dengue, apresentando um valor de $\rho=0,001$ (Correlação desprezível).

\section{Taxa de Incidência de dengue}

A taxa de incidência da dengue para Maceió (Figura 07) apresentou comportamento diferente no período analisado (2008-2015). Nos anos de 2009 e 2011, as taxas foram classificadas como de média incidência, apresentando os valores de 263/100 mil hab. e 267/100 mil hab. respectivamente. Os demais anos foram classificados como de alta incidência. A maior taxa de incidência da dengue ocorreu em 2010 (2.187/100 mil hab.), representando um aumento dos casos da dengue de $830 \%$ em relação ao ano anterior.

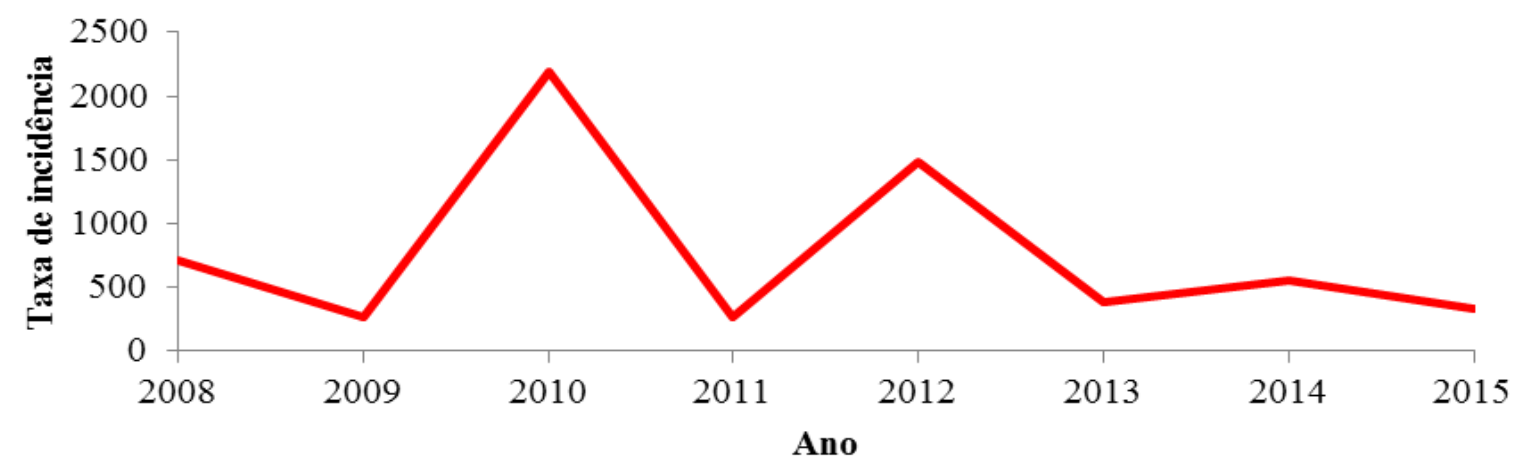

Coeficiente de Incidência de Dengue

Figura 07- Taxa de Incidência de Dengue para Maceió (2008-2015).

A taxa de incidência da dengue para João Pessoa (Figura 08) apresentou variável dentro do período analisado. No ano de 2009 foi classificado como de baixa incidência da doença, apresentando o valor de 19/100 mil hab. Os anos de 2008 e 2010 foram avaliados como de média incidência, as taxas apresentaram respectivamente os valores de 106/100 mil hab. e 153/100 mil hab. Os anos a partir de 2011 a 2015 foram classificados como de alta 
incidência, o qual o ano de 2012 apresentou a maior taxa de incidência da dengue, cerca de 700/100 mil hab.

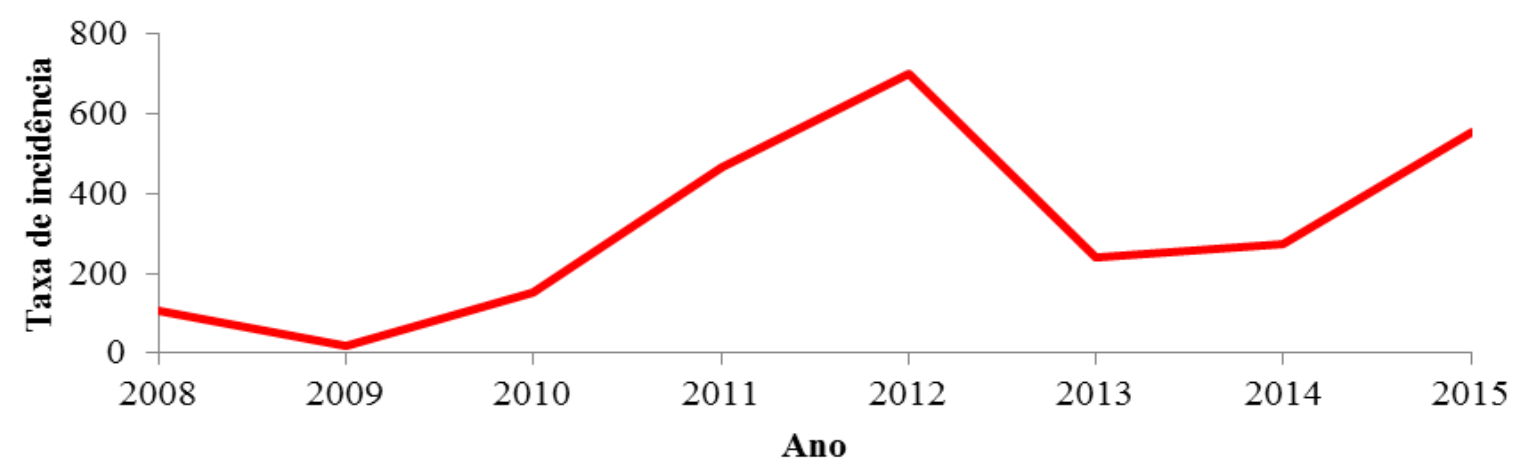

Coefieciente de Incidência de Dengue

Figura 08- Taxa de Incidência de Dengue para João Pessoa (2008-2015).

Para Salvador, A taxa de incidência da dengue (Figura 09) apresentou-se distinto dentro do período de análise. Os anos de 2008 e 2013 foram classificados como de baixa incidência, apresentando respectivamente os valores de 93/100 mil hab. e 69/100 mil hab. Os demais anos foram avaliados como de média incidência, em que as maiores incidências foram encontradas nos anos de 2009, 2010 e 2011, valores que oscilaram de 229 a 240/100 mil hab. Salvador não apresentou picos de alta incidência de dengue como as outras cidades demonstraram.

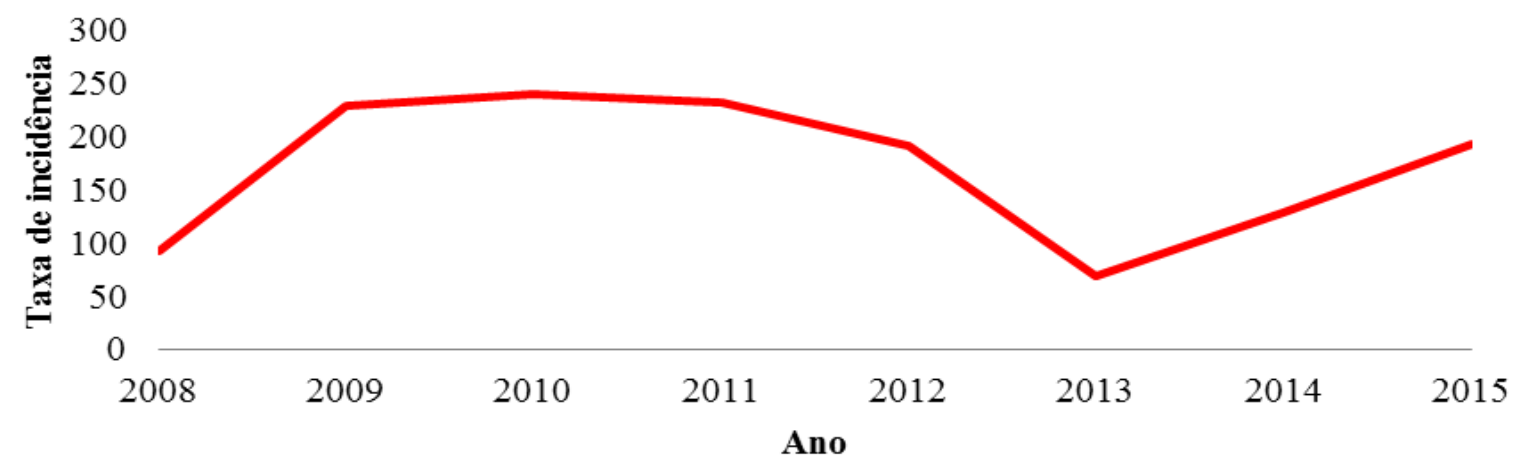

Coefíciente de incidência de dengue

Figura 09- Taxa de Incidência de Dengue para Salvador (2008-2015).

\section{CONCLUSÃO}

Através das análises dos gráficos que correlacionaram a precipitação média e o número de registro de casos de dengue elaborado para as três cidades no período de 2008 a 2015, observou-se uma relação direta entre essas duas variáveis, no qual os registros de casos 
de dengue seguiram a mesma tendência da precipitação média. Uma vez que, quando ocorre um aumento no volume das chuvas, os registros de casos de dengue aumentam, ou seja, a precipitação foi o fator preponderante para a manutenção da doença.

Ressaltando que, o conhecimento do período chuvoso, bem como o período seco de cada região é essencial para um planejamento eficiente no combate do foco do mosquito, evitando surtos de dengue devido ao acumulo de água parada nas épocas chuvosas.

Quando comparado às médias de temperatura do ar e os registros de casos de dengue, não foi observado um padrão coerente entre essas variáveis, porém a costa leste do Nordeste onde localizam-se as cidades em estudo, possui temperaturas elevadas durante o ano todo, clima favorável ao desenvolvimento do vetor da dengue a praticamente qualquer época do ano.

Na aplicação da correlação de Pearson, as cidades de Salvador e Maceió obtiveram forte correlação entre as variáveis precipitação média e casos de dengue e moderada para a cidade de João Pessoa. No entanto, as correlações entre a temperatura média do ar e os casos de dengue foram desprezíveis para todas as cidades analisadas.

Com o resultado do taxa de incidência de dengue foi possível ter uma perspectiva de como os casos confirmados de dengue variaram nas três cidades analisadas para o período de 2008 a 2015. Maceió, no geral, obteve os maiores índices, no qual nenhum dos anos apresentou baixa incidência de dengue. Já Salvador apresentou os menores índices, não exibindo índices de alta incidência de dengue.

\section{REFERÊNCIAS}

ARAUJO, A.C.; SILVA, J.P.; CUNHA, J.L.X.L. ARAUJO, J.L.O. Caracterização sócioeconômico-cultural de raizeiros e procedimentos pós-colheita de plantas medicinais comercializadas em Maceió, AL. Revista brasileira de plantas medicinais, Botucatu, v. 11, n. 1, p. 84-91, 2009

ARAúJO, V. E. M. D., BEZERrA, J. M. T., AMÂNCIO, F. F., PASSOS, V. M. D. A., CARNEIRO, M. . Aumento da carga de dengue no Brasil e unidades federadas, 2000 e 2015: análise do Global Burden of Disease Study 2015. Revista Brasileira de Epidemiologia, v.20, p.205-216, 2017.

BARBOSA, M. D. G. R., BAVIA, M. E; DA SILVA, C. E. P.; BARBOSA, F. R. Aspectos epidemiológicos dos acidentes escorpiônicos em Salvador, Bahia, Brasil. Ciência animal brasileira, v. 4, n. 2, p. 155-162, 2003. 
BESERRA, E.B.; CASTRO JR.; F.P.; SANTOS, J.W.; SANTOS, T.; FERNANDES, C.R.M. Biologia e Exigências Térmicas de Aedes aegypti (L.) provenientes de quatro Regiões Bioclimáticas da Paraíba. Neotropical Entomology, v.35, p. 853-860, 2006.

BRAGA, I.A.; VALLE, D. Aedes Aegypti: histórico do controle no Brasil. Epidemiol Serv Saude, v.16, p.113-118, 2007.

BRASIL. Ministério da Saúde. Dados situação epidemiológica Dengue. http://portalarquivos2.saude.gov.br/images/pdf/2018/agosto/21/Publicacao-BE-2018-SE30.pdf: Agos/2018.

CÂMARA, F.P.;THEOPHILO, R.L.G.;SANTOS,G.T.;PEREIRA, S.R.G.;CÂMARA,D.C.P.; MATOS, R.R.C. Estudo retrospectivo (histórico) da dengue no Brasil: características regionais e dinâmicas. Revista da Sociedade brasileira de medicina tropical, vol 40, Uberarba, 2007.

COSTA, I.M.P.; CALADO, D.C. Incidência dos casos de dengue (2007-2013) e distribuição sazonal de culicídeos (2012-2013) em Barreiras, Bahia. Epidemiol.Serv.Saude, v.25, p. 735744, 2016.

COSTA, M.S.; LIMA, L.C.; COSTA, J.M.; ZANELLA, M.E. Geografia da Saúde: pressupostos teóricos e o estudo da relação da precipitação com os casos de dengue no município de Eusébio-CE. Revista Geográfica de América Central, p.1-13, 2011.

DANTAS, R. T.; LIMEIRA, R.C.; ALENCAR, H.E. Influencia de variáveis meteorológicas sobre a incidência de dengue em João Pessoa-PB. Revista Fafibe On-line, Bebedouro, v. 3, p. 1-6, 2007.

FERREIRA A.F.; CHIARAVAlloti, F.; MONDINI, A. Dengue em Araraquara, SP: Epidemiologia clima e infestação por Aedes Aegypti. Revista de Saúde Pública. V. 52, p.1$10,2018$.

FLEXA, G. G.; CUNHA, F.A.; CUNHA, A.C. Relação da precipitação e casos de dengue notificados na baixada do Ambrósio, na área portuária de Santana/ AP, no ano de 2006. Revista de Humanidades do Curso de Ciências Sociais, $n^{\circ}$ 1, 2008.

Instituto Brasileiro de Geografia e Estatística - IBGE. População. 2017. [acessado em 10 ago. 2017]. Disponível em https://www.ibge.gov.br/apps/populacao/projecao/ 
PACHECO, I.C.S.; CARVALHO, A.M.A.; PONTES, E.R.J.C.; DA SILVA, M.G. Relação entre condições climáticas e incidência de dengue no município de Campo Grande, MS. Rev. Multitemas, v.22, p.235-252, 2017.

RIBEIRO, A.F.; MARQUES, G.R.A.M, VOLTOLINI, J.C; CONDINO, M.L. Associação entre incidência de dengue e as variáveis climáticas. Revista Saúde Pública, ed. 40, p.1-6, 2006.

SANTOS, G.S.; QUEIROZ, I.S.; GOLDFAB, M.; FIGUEIREDO, A.M.F. Incidência de Casos de Dengue na cidade de Sumé, Paraíba, Brasil, nos anos de 2009 a 2014. RSC online, v.5,p.5-17, 2016.

SOUSA, A. J. S ; OLIVEIRA, L. L. ; LUCAS, E. W. M. ; OLIVEIRA, L. L. Estudo comparativo entre a precipitação pluviométrica e o número de casos dengue notificados em belém-pa (1998-2003). Revista SODEBRAS, v. 1, p. 34-38, 2006.

VALADARES, A.F.; FILHO, J.R.C.; PELUZIO, J.M. Impacto da dengue em duas principais cidades do Estado do Tocantins: infestação e fator ambiental (2000 a 2010). Revista Epidemiologia e Serviços de Saúde, v. 22, Brasília, 2013.

VIANA, D.V.; IGNOTTI, E. A ocorrência da dengue e variações meteorológicas no Brasil: revisão sistemática. Rev Bras Epidemiol, v.16, p. 240-256, 2013. 